\title{
Panton-Valentine leukocidin and biofilm production of Staphylococcus aureus isolated from respiratory tract
}

\author{
Nilufer Cakır Aktas ${ }^{1}$, Zayre Erturan², Onur Karatuna $^{1}$, Aysegul Karahasan Yagci ${ }^{1}$ \\ ${ }^{1}$ Department of Clinical Microbiology, Marmara University, School of Medicine, Istanbul, Turkey \\ ${ }^{2}$ Department of Clinical Microbiology, Istanbul University, School of Medicine, Istanbul, Turkey
}

\begin{abstract}
Introduction: Staphylococcus aureus is one of the first bacteria colonizing in cystic fibrosis (CF) respiratory tract and different virulence factors are responsible for disease progression. It is not clear if CF S. aureus strains are more virulent than strains isolated from non-CF patients.

Methodology: Biofilm production was detected by a modified tissue culture plate method, presence of genes encoding for Panton-Valentine leukocidin (PVL) was investigated by a signal amplified sandwich hybridization assay and antimicrobial susceptibility patterns were detected by disk diffusion method.

Results: Staphylococcus aureus clinical isolates $(n=88)$ recovered from respiratory tract specimens in which 31 of them were from cystic fibrosis (CF) patients were analysed. Biofilm production was detected in $96.8 \%$ of CF isolates in which $32.3 \%$ exhibited strong positive phenotype and in $47.4 \%$ of non-CF isolates in which strong positive phenotype was not observed $(\mathrm{p}<0.05)$. All CF isolates were methicillin susceptible, whereas $53.4 \%$ of non-CF isolates $(n=31)$ were methicillin resistant. No resistance was observed for vancomycin, chloramphenicol and trimethoprim/sulfamethoxazole in any of the isolates. PVL genes were detected only in two isolates (2.3\%), one from each group, $\mathrm{CF}$ and non-CF, which both were methicillin susceptible

Conclusion: Biofilm rather than PVL production appears to be an important virulence factor in CF patients.
\end{abstract}

Key words: Cystic fibrosis; Panton-Valentine leukocidin; antimicrobial; biofilm

J Infect Dev Ctries 2013; 7(11):888-891. doi:10.3855/jidc.4135

(Received 15 August 2013 - Accepted 09 October 2013)

Copyright $(C 2013$ Aktas et al. This is an open-access article distributed under the Creative Commons Attribution License, which permits unrestricted use, distribution, and reproduction in any medium, provided the original work is properly cited.

\section{Introduction}

Impairment of mucociliary transport and the cleaning function of upper airways related to recurrent and chronic colonization with bacterial pathogens, such as Staphylococcus aureus, Haemophilus influenzae, and Pseudomonas aeruginosa are among the most important causes of morbidity and mortality in cystic fibrosis (CF) patients [1].

$S$. aureus is one of the first bacteria colonizing in $\mathrm{CF}$ respiratory tract and particularly methicillin resistant strains have been associated with worse prognosis [2]. In general, antibiotic resistance of bacteria in respiratory infections of CF patients is of increasing concern and resistance monitoring is important for early treatment of the patients. Biofilm formation was demonstrated for bacterial pathogens in $\mathrm{CF}$ and provides an advantage to the bacteria for chronic colonization and making eradication with antimicrobials difficult [3]. Panton-Valentine leukocidin (PVL) is an exoprotein produced by $S$. aureus which kills leukocytes, causes severe tissue destruction and necrosis associated with community acquired pneumonia [4]. Gillet et al. [5] reported that PVL-producing $S$. aureus strains caused rapidly progressive, haemorrhagic, necrotizing pneumonia with a high mortality rate, mainly in immunocompetent children and young adults. PVL positive methicillin resistant $S$. aureus (MRSA) strains were associated with development of invasive lung infections including lung abscess in CF patients [6]. We compared biofilm production capabilities, presence of PVL genes and antimicrobial susceptibility properties in $S$. aureus isolates recovered from respiratory specimens of $\mathrm{CF}$ and non-CF patients.

\section{Methodology}

$S$. aureus strains isolated from respiratory specimens such as sputum, deep tracheal aspirates (DTA) and bronchoalveolar lavage materials were collected from hospitalized patients with lower respiratory tract infection symptoms $(\mathrm{n}=57)$, whereas 
DTA samples were taken from patients with CF ( $\mathrm{n}=$ 31) followed in Cytic Fibrosis Center of Istanbul University during 2008-2010. Isolates were identified by colony morphology, Gram stain, catalase, tubecoagulase, DNase tests and final identification was performed with Vitek 2 automated identification system (bioMérieux, l'Etoile, France). The isolates were stored in trypticase soy broth supplemented with $15 \%$ glycerol at $-80^{\circ} \mathrm{C}$ and subcultured onto trypticase soy agar supplemented with 5\% sheep blood agar for experiments.

A modified tissue culture plate method was used for detection of biofilm formation [7]. We used trypticase soy broth (Difco, Franklin Lakes, USA) treated with $0.1 \mathrm{M}$ ethylene diamine tetra-acetic acid (EDTA) to limit iron and $5 \mathrm{ml}$ of the medium was inoculated with $S$. aureus isolates and incubated 24 hours at $37^{\circ} \mathrm{C}$. Next, 1:100 dilution of each isolate was inoculated into fresh TSB-EDTA medium in microplates. After 24 hour of incubation at $37^{\circ} \mathrm{C}$, plates were washed three times with phosphate buffered saline, left to dry for one hour at room temperature and then stained with $0.2 \mathrm{ml}$ of safranin $\mathrm{O}$ $\left(1 \% \mathrm{w} / \mathrm{v}\right.$ in $\left.\mathrm{H}_{2} \mathrm{O}\right)$. Excess stain was washed with tap water and the optical density of the biofilm was determined by absorbance read at $490 \mathrm{~nm}$ in a microplate reader and values were obtained from three independent experiments. Staphylococcus epidermidis ATCC 35894 was used as positive control, $S$. epidermidis ATCC 12228 as negative control. The evaluation of clinical strains for biofilm production ability was accomplished using the difference between the mean absorbance values obtained from positive and negative control strains. Strains with absorbance values $<20 \%$ of the difference were evaluated as negative for biofilm production. Strains with absorbance values between $\geq 20 \%-<40 \%, \geq 40 \%-<$ $70 \%$ and $\geq 70 \%$ of the difference were regarded as weak positive, moderate positive and strong positive, respectively.

The correlation between the biofilm production ability of CF and non-CF isolates was evaluated using SPSS software (SAS Institute, Cary, USA) with Fisher's exact test and $p<0.05$ was considered statistically significant.

The susceptibility of the isolates was determined by disk diffusion method in accordance with Clinical and Laboratory Standards Institute (CLSI) standards [8]. S. aureus ATCC 25923 was used as the control strain. The antibiotics tested were penicillin, erythromycin, gentamicin, clindamycin, vancomycin, rifampin, ciprofloxacin, chloramphenical, trimethoprim-sulfamethoxazole and tetracycline. Susceptibility testing for methicillin was carried out using cefoxitin $(30 \mu \mathrm{g})$ and oxacillin $(1 \mu \mathrm{g})$ disks.

PVL EVIGENE (AdvanDx, Vedbaek, Denmark), a signal amplified sandwich hybridization assay for its ability to detect $l u k \mathrm{~S}-\mathrm{PV}$ and $l u k \mathrm{~F}-\mathrm{PV}$, the genes encoding PVL in $S$. aureus, was used according to manufacturer's instructions. S. aureus ATCC 49775 PVL and S. aureus LY19990053 were used as positive controls. The test is performed in microwells containing capture probes for the $l u k \mathrm{~S}-l u k \mathrm{~F}-\mathrm{PV}$ genes. If the $l u k \mathrm{~S}-l u k \mathrm{~F}-\mathrm{PV}$ genes are present, capture probe lukS-lukF-PV genes - detection probe complexes are formed. These complexes are subsequently visualized by a conjugate-substrate signal amplification reaction. Samples with the lukS-lukF-PV genes produced a red solution in the assay and results were evaluated visually.

\section{Results}

Biofilm production was detected in $96.8 \%$ of $\mathrm{CF}$ isolates in which $32.3 \%$ exhibited strong positive phenotype and in $47.4 \%$ of non-CF isolates in which none of the isolates exhibited strong positive phenotype $(\mathrm{p}<0.05)$ (Table 1).

All CF isolates were methicillin susceptible, whereas $53.4 \%$ of non-CF isolates were methicillin resistant. No resistance was observed for vancomycin, chloramphenicol and trimethoprim/sulfamethoxazole in any of the isolates. Methicillin susceptible strains from both groups, CF $(n=31)$ and non-CF $(n=26)$, showed similar antimicrobial resistance rates to tested antimicrobials (Table 2). Although biofilm production rate was notably higher in $\mathrm{CF}$ isolates compared to non-CF isolates $(96.8 \%$ vs. $46.2 \%)$, a correlation between biofilm production and antimicrobial resistance was not observed.

PVL genes were detected only in two of the isolates $(2.3 \%)$, one isolate from $\mathrm{CF}$ group and one from non-CF, which both were methicillin susceptible.

\section{Discussion}

Chronic lung disease is the most serious clinical expression of $\mathrm{CF}$ and the progressive lung injury associated with endobronchial infection, usually first with $S$. aureus and then with Pseudomonas aeruginosa, eventually leads to death from respiratory failure [9]. The clinical significance of colonization/infection with $S$. aureus is variable, 
Table 1. Results of biofilm forming ability studies in CF* and non-CF Staphylococcus aureus isolates.

\begin{tabular}{lcccc}
\hline & \multicolumn{4}{c}{ Biofilm forming ability } \\
\cline { 2 - 5 } CF isolates $(\mathrm{n}=31)$ & Negative & Weak positive & Moderate positive & Strong positive \\
Non-CF isolates $(\mathrm{n}=57)$ & $1(3.2 \%)$ & $8(25.8 \%)$ & $12(38.7 \%)$ & $10(32.3 \%)$ \\
\hline Total $(\mathrm{n}=88)$ & $30(52.6 \%)$ & $14(24.6 \%)$ & $22.8 \%)$ & 0 \\
\hline$*$ CF: cystic fibrosis & $31(35.2 \%)$ & $22(25.0 \%)$ & $25(28.4 \%)$ & $10(11.4 \%)$ \\
\hline
\end{tabular}

Table 2. The relationship between biofilm forming ability and resistance to antimicrobials in methicillin susceptible Staphylococcus aureus isolates recovered from $\mathrm{CF}^{*}$ and non-CF patients.

\begin{tabular}{|c|c|c|c|c|}
\hline \multirow{3}{*}{ Resistance to antimicrobial agents } & \multicolumn{4}{|c|}{ Biofilm forming ability } \\
\hline & \multicolumn{2}{|c|}{$C F$ isolates $(n=31)$} & \multicolumn{2}{|c|}{ Non-CF isolates $(n=26)$} \\
\hline & $\begin{array}{l}\text { positive } \\
(\mathrm{n}=30)\end{array}$ & $\begin{array}{c}\text { negative } \\
(\mathbf{n}=1) * *\end{array}$ & $\begin{array}{l}\text { positive } \\
(\mathrm{n}=12)\end{array}$ & $\begin{array}{c}\text { negative } \\
(\mathrm{n}=14)\end{array}$ \\
\hline Erythromycin & $8(26.7 \%)$ & 0 & $1(8.3 \%)$ & $2(14.3 \%)$ \\
\hline Clindamycin & $1(3.3 \%)$ & 0 & 0 & 0 \\
\hline Tetracycline & $5(16.7 \%)$ & 1 & 0 & $2(14.3 \%)$ \\
\hline Ciprofloxacin & $3(10 \%)$ & 0 & $1(8.3 \%)$ & $1(7.1 \%)$ \\
\hline Rifampin & $1(3.3 \%)$ & 0 & 0 & 0 \\
\hline Vancomycin & 0 & 0 & 0 & 0 \\
\hline Chloramphenicol & 0 & 0 & 0 & 0 \\
\hline
\end{tabular}

* CF: cystic fibrosis, ** For columns with less than ten total isolates, no percentage is indicated

leading to numerous regimens for prevention and treatment of patients colonized with this pathogen. The independent and additive impacts of $S$. aureus on lower airway disease increase lower airway inflammation in infants and young children with $\mathrm{CF}$ and the presence of both $S$. aureus and P. aeruginosa could demonstrate significant additive effect on the different measures of lower airway inflammation [10]. Despite increasing frequency of isolation and potential serious morbidity, very little is known about the characteristics of the virulence of $S$. aureus in $\mathrm{CF}$ patients.

Toxic effect of PVL results from synergistic action of two transcribed genes, $l u k \mathrm{~S}-\mathrm{PV}$ and $l u k \mathrm{~F}-\mathrm{PV}$ and presence of these genes was demonstrated in two of our isolates which were both methicillin susceptible. This result is similar with findings of Molina et al. [11] who detected PVL gene in any of the 98 MRSA$\mathrm{CF}$ isolates but described a multiresistant biofilm forming clone in most of the patients. In a period between 2005 and 2007, 64 CF patients in Italy were screened and PVL was detected in only 2 isolates [12]. Elizur et al. [6] described the emergence of $\mathrm{PVL}^{+}$ MRSA infection in $15 \%$ of $\mathrm{CF}$ patients and demonstrated that the patients who acquired a $\mathrm{PVL}^{+}$ organism were more likely to have a focal pulmonary infiltrate on chest radiograph, a markedly greater decline in $\mathrm{FEV}_{1}$ suggesting that early identification and treatment of $\mathrm{CF}$ patients with newly acquired $\mathrm{PVL}^{+}$MRSA may be crucial.

We detected a higher and stronger biofilm production in $\mathrm{CF}$ patients than in non-CF patients. Cafiso et al. [12] also stated that all strains isolated from CF patients were medium or strong biofilm producers and this result seemed to be consistent with the ability of these microorganisms to adhere and persist in the lungs of these patients.

Although data from the American CF Foundation Patient Registry shows that the prevalence of MRSA infection in $\mathrm{CF}$ has increased from $6.1 \%$ in 2001 to $23.7 \%$ in 2009 , all of our CF isolates were methicillin susceptible, also presenting low level of resistance to non- $\beta$-lactam antimicrobials [13]. In Brazil, $7 \%$ of CF patients were found colonized by MRSA, whereas none were positive for PVL [14].

We could not detect significant difference of resistance among biofilm positive and negative strains 
in our study. It was claimed that antimicrobial susceptibility results determined for planktonic cultures do not provide sufficient information on the efficacy of antimicrobials against infections involving biofilms and that in vivo conditions could be different and antimicrobial activity could be diminished. Biofilm formation in $S$. aureus is coordinated by quorum sensing (QS), a cell-cell communication system and RNAIII-activating protein (RAP) and its target molecule (TRAP) is the best studied system [15]. QS inhibitors were shown to affect later stages of biofilm formation and detachment of Burkholderia cepacia complex strains as another opportunistic pathogens causing life-threatening infections in $\mathrm{CF}$ patients, which are resistant to many antimicrobial agents [16]. Extracts from three medicinal plants (Ballota nigra, Castanea sativa, and Sambucus ebulus) exhibited anti-QS activity in a pathogenic MRSA isolate [17]. Although our CF isolates were susceptible to tested antimicrobials, QS inhibitors could be an alternative for treatment of $S$. aureus infections in $\mathrm{CF}$ patients, considering increasing levels of antimicrobial resistance of the bacteria.

\section{References}

1. Valenza G, Tappe D, Turnwald D, Frosch M, König C, Hebestreit H, Abele-Horn M (2008) Prevalence and antimicrobial susceptibility of microorganisms isolated from sputa of patients with cystic fibrosis. J Cyst Fibros 7: 123127.

2. Renders NH, van Belkum A, Overbeek SE, Mouton JW, Verbrugh HA (1997) Molecular epidemiology of Staphylococcus aureus strains colonizing the lungs of related and unrelated cystic fibrosis patients. Clin Microbiol Infect 3: 216-221.

3. Jackson K, Keyser R, Wozniak DJ (2003) The role of biofilms in airway disease. Semin Respir Crit Care Med 24: 663-670.

4. Lina G, Piémont Y, Godail-Gamot F, Bes M, Peter MO, Gauduchon V, Vandenesch F, Etienne J (1999) Involvement of Panton-Valentine leukocidin-producing Staphylococcus aureus in primary skin infections and pneumonia. Clin Infect Dis 29: 1128-1132.

5. Gillet $Y$, Issartel B, Vanhems $P$, Fournet JC, Lina G, Bes M, Vandenesch F, Piémont Y, Brousse N, Floret D, Etienne J (2002) Association between Staphylococcus aureus strains carrying gene for Panton-Valentine leukocidin and highly lethal necrotising pneumonia in young immunocompetent patients. Lancet 359: 753-759.

6. Elizur A, Orscheln RC, Ferkol TW, Atkinson JJ, Dunne WM Jr, Buller RS, Armstrong JR, Mardis ER, Storch GA, Cannon CL (2007) Panton-Valentine Leukocidin-positive methicillinresistant Staphylococcus aureus lung infection in patients with cystic fibrosis. Chest 131: 1718-1725.
7. Mathur T, Singhal S, Khan S, Upadhyay DJ, Fama T, Rattan A (2006) Detection of biofilm formation among the clinical isolates of staphylococci: an evaluation of three different screening methods. Indian J Med Microbiol 24: 25-29.

8. CLSI - Clinical and Laboratory Standards Institute (2008) Performance Standards for Antimicrobial Susceptibility Testing; Sixteenth Informational Supplement. CLSI document M100-S16. Clinical and Laboratory Standards Institute Wayne, PA, USA.

9. Sagel SD, Gibson RL, Emerson J, McNamara S, Burns JL, Wagener JS, Ramsey BW; Inhaled Tobramycin in Young Children Study Group; Cystic Fibrosis Foundation Therapeutics Development Network. (2009) Impact of Pseudomonas and Staphylococcus infection on inflammation and clinical status in young children with cystic fibrosis. J Pediatr 154: 183-188.

10. Armstrong DS, Grimwood K, Carlin JB, Carzino R, Gutièrrez JP, Hull J, Olinsky A, Phelan EM, Robertson CF, Phelan PD (1997) Lower airway inflammation in infants and young children with cystic fibrosis. Am J Respir Crit Care Med 156: 1197-1204.

11. Molina A, Del Campo R, Máiz L, Morosini MI, Lamas A, Baquero F, Cantón R (2008) High prevalence in cystic fibrosis patients of multiresistant hospital-acquired methicillin-resistant Staphylococcus aureus ST228-SCCmecI capable of biofilm formation. J Antimicrob Chemother 62: 961-967.

12. Cafiso V, Bertuccio T, Spina D, Campanile F, Bongiorno D, Santagati M, Sciacca A, Sciuto C, Stefani S (2010) Methicillin resistance and vancomycin heteroresistance in Staphylococcus aureus in cystic fibrosis patients. Eur J Clin Microbiol Infect Dis 29: 1277-1285.

13. Cystic Fibrosis Foundation (2010) Cystic Fibrosis Foundation Patient Registry: Annual Data Report 2009. Bethesda, MD, USA.

14. Mimica MJ (2012) Staphylococcus aureus and cystic fibrosis. J Cyst Fibros 11: 71.

15. Balaban N, Goldkorn T, Gov Y, Hirshberg M, Koyfman N, Matthews HR, Nhan RT, Singh B, Uziel O (2001) Regulation of Staphylococcus aureus pathogenesis via target of RNAIIIactivating Protein (TRAP). J Biol Chem 276: 2658-2667.

16. Brackman G, Hillaert U, Van Calenbergh S, Nelis HJ, Coenye $\mathrm{T}$ (2009) Use of quorum sensing inhibitors to interfere with biofilm formation and development in Burkholderia multivorans and Burkholderia cenocepacia. Res Microbiol 160: 144-151.

17. Quave CL, Plano LR, Bennett BC (2011). Quorum sensing inhibitors of Staphylococcus aureus from Italian medicinal plants. Planta Med 77: 188-195.

\section{Corresponding author}

Professor Aysegul Karahasan Yagci, MD

Marmara University, School of Medicine,

Department of Clinical Microbiology

34668, Haydarpasa, Istanbul

Turkey

Fax: $902164144732 \quad$ Tel: 905325823582

E- mail: ayagci@marmara.edu.tr

Conflict of interests: No conflict of interests is declared. 